\title{
Identifying a low-frequency oscillation in Galileo IOV pseudorange rates
}

\author{
Daniele Borio $\cdot$ Ciro Gioia $\cdot$ Neil Mitchison
}

Received: 29 August 2014/Accepted: 5 February 2015/Published online: 24 February 2015

(C) The Author(s) 2015. This article is published with open access at Springerlink.com

\begin{abstract}
Galileo, the European global navigation satellite system, is in its in-orbit validation phase and the four satellites which have been available for some months now have allowed a preliminary analysis of the system performance. Previous studies have showed that Galileo will be able to provide pseudorange measurements more accurate than those provided by GPS. However, a similar improvement was not found for pseudorange rate observations in the velocity domain. This fact stimulated additional analysis of the velocity domain, and, in particular, an unintended oscillatory component was identified as the main error source in the velocity solution. The magnitude of such oscillation is less than $10 \mathrm{~cm} / \mathrm{s}$, and its period is in the order of few minutes. A methodology was developed to identify oscillatory components in the Galileo IOV pseudorange rate observables, and it was verified that the measurements from Galileo IOV PFM and Galileo IOV FM2 are affected by a small oscillatory disturbance. This disturbance stems from the architecture adopted for combining the frequency references provided by the two active clocks present in the Galileo satellites. The issue has been solved in Galileo IOV FM3 and Galileo IOV FM4, and the oscillatory component has been eliminated. We also propose a methodology for removing this unwanted component from the final velocity solution and for determining
\end{abstract}

\footnotetext{
D. Borio $(\bowtie) \cdot$ C. Gioia $\cdot$ N. Mitchison

Security Technology Assessment (STA) Unit, Joint Research Centre (JRC), Institute for the Protection of the Citizen (IPSC), European Commission, Ispra, VA, Italy

e-mail: daniele.borio@jrc.ec.europa.eu

C. Gioia

e-mail: ciro.gioia@jrc.ec.europa.eu

N. Mitchison

e-mail: Neil.MITCHISON@ec.europa.eu
}

the performance that Galileo will be able to achieve. The analysis shows that Galileo velocity solution will provide a root-mean-square error of about $8 \mathrm{~cm} / \mathrm{s}$ even in the limited geometry conditions achieved using only four satellites. This shows the potential of Galileo also in the determination of user velocity.

Keywords Galileo · In-orbit validation (IOV) . Oscillators · Velocity analysis

\section{Introduction}

Galileo, the European global navigation satellite system (GNSS), is in its validation phase, and four in-orbit validation (IOV) satellites allow the analysis of the system's stand-alone performance. Several research groups have investigated the performance of Galileo positioning both in single point (SP) mode (Gioia et al. 2014) and using a real-time kinematic (RTK) approach (Odijk et al. 2014). The potential of Galileo has been clearly established, and, in particular, it has been shown that Galileo will be able to provide pseudorange measurements more accurate than those from GPS. However, a similar improvement was not found for pseudorange rate observations and consequently in the velocity estimation (Gioia et al. 2014). This result motivated the additional analysis described here, where the IOV pseudorange rate observations and Galileo stand-alone velocity solution are further studied.

The analysis showed that small oscillatory disturbances are present in the velocity solution computed for a static Galileo receiver. Moreover, the study of the different components used for velocity computation indicated that the sources of such oscillations are the Galileo pseudorange rate measurements. For this reason, a methodology was 
developed to identify oscillatory components in the Galileo IOV pseudorange rate observables, and it was shown that only the measurements from Galileo IOV PFM and Galileo IOV FM2 are affected by this disturbance.

The presence of small oscillatory components in the pseudorange rate measurements from Galileo IOV PFM and Galileo IOV FM2 has been verified using a modified experimental setup involving two Galileo-capable receivers. In this way, it was possible to obtain synchronous measurements from two independent sources, and the phenomenon was observed in the measurements from both receivers. Finally, high rate data from the International GNSS Service (IGS) (Dow et al. 2009) were used, and the analysis was repeated on pseudorange rate measurements from IGS station WTZ3, from Wettzell, Germany. Also in this case, oscillatory components were clearly observed. Moreover, the analysis of datasets collected in different days proved that the centre frequency of the oscillatory component is time varying.

The presence of small oscillatory components in the measurements from Galileo IOV PFM and Galileo IOV FM2 was discussed during the IOV review event organized by the European Space Agency (ESA) in October 2013. From the discussion, it emerged that the disturbances observed are due to the approach adopted for combining the frequency references produced by the onboard clocks of the Galileo IOV PFM and Galileo IOV FM2 satellites. Galileo is the first GNSS which will use several on-board clocks for the generation of time and frequency reference signals (Rochat et al. 2012). In particular, two passive hydrogen masers (PHMs) and two rubidium frequency standards (RFSs) clocks are installed on the IOV satellites (Rochat et al. 2012). The PHMs are used as primary clocks, whereas the RFSs are activated in case of failure of the primary devices. Thus, two frequency references are available at any time, and a combining algorithm is needed to generate a single timing signal. The first version of the combining algorithm generates a spurious oscillation at the frequency of about $6 \mathrm{~Hz}$. When the measurements are taken at a frequency of $1 \mathrm{~Hz}$, the spurious oscillation is aliased back to a very low frequency. The disturbances observed are the result of this aliased spurious oscillation.

The identification of disturbances in the measurements is fundamental for the correct interpretation of the results obtained using the products provided by GNSS satellites. For example, Benton and Mitchell $(2012,2014)$ showed that phase measurements from IIR GPS satellites are sporadically affected by clock anomalies which could be interpreted as strong ionospheric events. The analysis provided in (Benton and Mitchell 2012, 2014) allows the exclusion of ionospheric phenomena and the correct interpretation of the experimental results obtained using
GNSS observables. The analysis presented here allows the correct interpretation of the results obtained using Galileo pseudorange rate measurements.

The oscillatory disturbances are not present in Galileo IOV FM3 and Galileo IOV FM4: Future Galileo satellites will be able to fully exploit the benefits brought by the Galileo atomic clocks. For this reason, Galileo performance should be analyzed in the absence of such oscillatory components. With this in mind, the velocity analysis performed by Gioia et al. (2014) has been extended, and a methodology based on a two pole notch filter (Borio et al. 2008) has been suggested for removing such disturbances from the velocity solution. The analysis performed in (Gioia et al. 2014) has been repeated using filtered velocity components, and the potential of the Galileo system has been proven also with respect to the determination of the user velocity.

Although the E1 signal is considered for the experimental analysis, oscillatory components can be also observed in measurements from other frequencies.

\section{Experimental setup and velocity computation}

In this section, the experimental setups used to collect Galileo observables are described along with the algorithm adopted for the velocity computation.

Two different setups were adopted in order to perform different types of experiments. The methodology described in (Gioia et al. 2014) was initially adopted for the pseudorange rate and velocity analysis. A Javad RingAnt-G3T was mounted on the roof top of the European Microwave Signature Laboratory (EMSL) in the Joint Research Centre (JRC) premises in Ispra, Italy. The position of the antenna was carefully selected in order to minimize multipath effects. The antenna was then connected to a Septentrio PolarRxS receiver able to simultaneously collect GPS, GLONASS, and Galileo measurements on several GNSS bands. The position of the antenna was carefully surveyed using double difference carrier phase positioning. This information was used to compute the user velocity as described below. With this calibrated setup, it was possible to collect several days of data which were used for the characterization of Galileo observables as discussed by Gioia et al. (2014).

A second setup involving two Galileo-capable receivers was then used to validate and further investigate the results obtained with the Septentrio PolarRxS receiver. The use of a second receiver allows one to verify that the oscillations observed were not artifacts created by the Septentrio PolarRxS receiver or by other local effects.

In particular, the experimental setup was modified according to the diagram depicted in Fig. 1: A second 


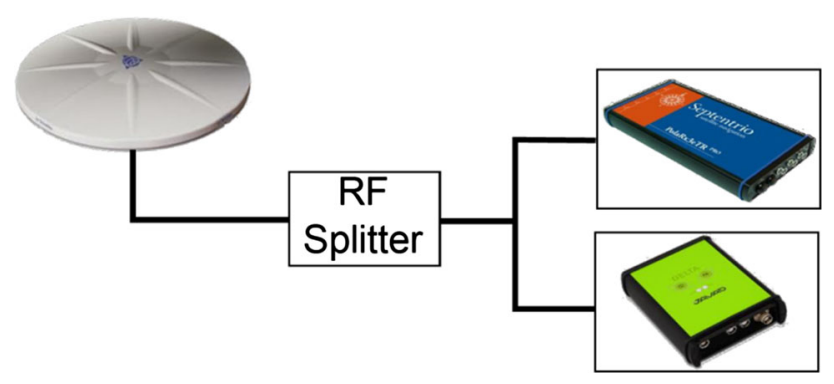

Fig. 1 Modified experimental setup involving a second Galileocapable receiver. The two receivers were connected to the same antenna through a RF splitter

Galileo-capable receiver, a Javad Delta-3 was connected to the roof-top antenna through a radio frequency (RF) splitter. Moreover, the roof-top antenna, a Javad RingAntG3T, used for the first series of experiments was replaced by a Trimble Zephyr geodetic antenna, to exclude any possibility of artifacts arising from the receiver antenna.

These two setups were used to collect Galileo measurements and compute the velocity solution according to the methodology detailed below. In particular, GNSS receivers are able to provide Doppler measurements representing the frequency shift produced by the satellite/ receiver relative motion (Kaplan and Hegarty 2006). Using Doppler observables, GNSS receivers are able to compute the three-dimensional user velocity; the algorithm adopted here for velocity estimation is developed in the East, North, Up (ENU) frame.

The received frequency, $f_{\mathrm{R}}$ expressed in $(\mathrm{Hz})$, is related to the transmitted one, $f_{\mathrm{T}}$, by the Doppler equation (Kaplan and Hegarty 2006):

$f_{\mathrm{R}}=f_{\mathrm{T}}\left(1-\frac{\left(\underline{v}_{\mathrm{S}}-\underline{v}_{\mathrm{R}}\right) \cdot \underline{a}}{c}\right)$

where $\underline{v}_{S}$ and $\underline{v}_{R}$ are the vectors containing the satellite and receiver velocity, respectively, $\underline{a}$ is the unit vector pointing along the line of sight from the user to the satellite. The underscore indicates the vectorial form of the elements. Equation (1) does not take into account the effects of the satellite and receiver clocks which are introduced below.

The Doppler shift due to the relative motion between satellite and receiver is given by:

$\Delta f=f_{\mathrm{R}}-f_{\mathrm{T}}=-f_{\mathrm{T}} \frac{\left(\underline{v}_{\mathrm{S}}-\underline{v}_{\mathrm{R}}\right) \cdot \underline{a}}{c}$

where the dot product, $\left(\underline{v}_{S}-\underline{v}_{R}\right) \cdot \underline{a}$, is the projection of the relative velocity vector along the receiver satellite direction, i.e., the range rate.

Thus, the expression of the range rate, $\dot{d}$, is obtained scaling the Doppler shift by the wavelength, $\lambda$

$\dot{d}=-\lambda \Delta f$.
The measured $\dot{d}(\mathrm{~m} / \mathrm{s})$ is affected by the receiver and satellite clock drifts, $c \dot{\delta} t_{\mathrm{S}}$ and $c \dot{\delta} t_{\mathrm{R}}$, so the measurement is called pseudorange rate and denoted by $\dot{\rho}$ :

$\dot{\rho}=\dot{d}-c \dot{\delta} t_{\mathrm{S}}+c \dot{\delta} t_{\mathrm{R}}$.

Different approaches can be adopted to compute the user velocity using pseudorange rates. In this analysis, we assume the receiver position as known. After correcting the term due to the satellite clock drift and replacing $\dot{d}=$ $\left(v_{\mathrm{S}}-v_{\mathrm{R}}\right) \cdot \underline{a}$ in (4), the following condition is obtained:

$\delta \dot{\rho}=-\underline{v}_{\mathrm{R}} \cdot \underline{a}+c \dot{\delta} t_{\mathrm{R}}$

where $\delta \dot{\rho}=\dot{\rho}-\underline{v}_{\mathrm{S}} \cdot \underline{a}$ is the difference between the measured and the predicted pseudorange rates.

A set of $N$ pseudorange rate measurements defines a system of $N$ equations which can be expressed in matrix form as:

$\delta \underline{\dot{\rho}}=H \underline{v}$

where $H$ is the design matrix, and $\underline{v}$ is the state vector which contains the receiver velocity and the receiver clock drift and is defined as

$\underline{v}=\left[\begin{array}{c}v_{\mathrm{E}} \\ v_{\mathrm{N}} \\ v_{\mathrm{U}} \\ c \dot{\delta} t_{\mathrm{R}}\end{array}\right]$.

Finally $\underline{v}$ can be estimated using a weighted least squares (WLS) estimator as

$\underline{v}=\left(H^{T} W H\right)^{-1} H^{T} W \delta \dot{\rho}$

where $W$ is the weighting matrix, representing the different accuracies of the measurements. The Doppler measurement accuracy is simply assumed to be inversely proportional to the sine of the satellite elevation.

\section{Pseudorange rate pre-filtering}

As discussed in the introduction, the oscillations observed in the velocity solution motivated a thorough analysis of the pseudorange rate measurements which are used for the velocity computation. It was verified that a direct inspection and a frequency domain analysis of the observables do not allow a clear identification of oscillatory components in pseudorange rate measurements. In particular, pseudorange rate variations are mainly due to the satellite motion (Hoffmann-Wellenhof et al. 1992). These variations are several orders of magnitude higher than the oscillations observed in the velocity domain. Over short time intervals, pseudorange rate variations due to satellite motion can be approximated by a polynomial curve and thus can be 
removed through filtering. For this reason, a pre-filtering stage was designed to remove these components.

The structure of the filter proposed is shown in Fig. 2. The filter is made of three components: a differentiator of order $K$, a compensator of order $K$, and a low-pass filter.

The differentiator is characterized by the following transfer function (Proakis and Manolakis 1995):

$H_{\mathrm{d}, K}(z)=\left(1-z^{-1}\right)^{K}$

which removes polynomial terms up to order $K-1$, since a polynomial of order $K-1$ has a $K$ th derivative equal to zero. Note that if an oscillatory component was present, it would be preserved by the filtering performed by (9).

The compensator is intended to ensure that the differentiator does not affect signal components far from the zero frequency; it is characterized by the transfer function

$H_{\mathrm{c}, k}=\frac{1}{\left(1-k_{\alpha} z^{-1}\right)^{K}} \quad 0<k_{\alpha}<1$

where $k_{\alpha}$ is the filter contraction factor (Borio et al. 2006). The combination of differentiator and compensator gives a notch filter (Borio et al. 2006) with a narrow notch around the zero frequency. Signal components far from the zero frequency are not affected by the filter, and the width of the filter notch is controlled by $k_{\alpha}$ (Borio et al. 2006). In the following, $k_{\alpha}=0.99$ is adopted.

Finally, a Butterworth filter of order 9 was used to reduce the impact of high frequency noise in the pseudorange rate observables. This filter component was used for noisy measurements, and it is not necessary for good quality measurements such as those presented here. The overall transfer function of the pre-filtering stage is given by:

$H_{n}(z)=\frac{\left(1-z^{-1}\right)^{K}}{\left(1-k_{\alpha} z^{-1}\right)^{K}} H_{\mathrm{B}}(z)$

where $H_{\mathrm{B}}(z)$ is the transfer function of the Butterworth filter used for the removal of high frequency noise.

An example of filtered pseudorange rate observables can be found in Fig. 3: An oscillatory component can be seen

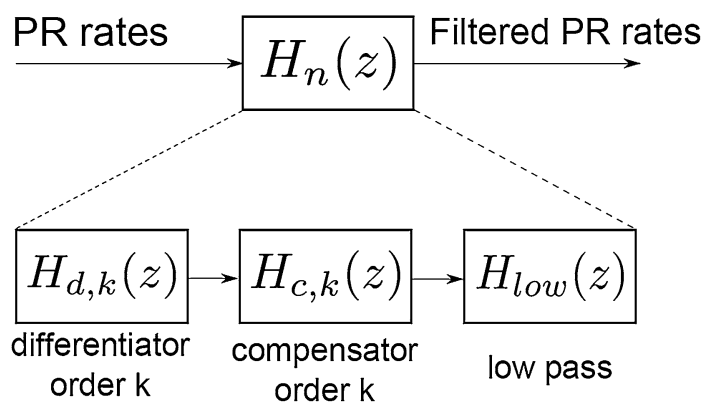

Fig. 2 Structure of the filter designed for pre-processing pseudorange rate measurements in the measurements from Galileo IOV PFM and Galileo IOV FM2. The measurements in Fig. 3 were collected during GPS week 1746 using the first setup described above. The pseudorange rate observations shown in Fig. 3 are further analyzed in the frequency domain in Fig. 4 which shows the PSDs of the filtered pseudorange rate measurements obtained from the four IOVs. The PSDs have been computed using Welch's periodogram (Stoica and Moses 2005).

The analysis provided in Fig. 4 confirms the presence of oscillatory components in the filtered pseudorange rate measurements from Galileo IOV PFM and Galileo IOV FM2. The two oscillatory components have approximately the same frequency which is

$f_{0}=0.0035 \mathrm{~Hz} \quad T_{0}=286 \mathrm{~s}$

$T_{0}$ is the period corresponding to such oscillation. The analysis also confirms that the filtered pseudorange rates from Galileo IOV FM3 and Galileo IOV FM4 do not show such behavior, indicating that these unwanted components only affect the first batch of IOV satellites.

It is noted that different approaches could have been used to isolate the oscillatory components described above. For example, the polynomial variations induced by the satellite motion could have been predicted from the satellite and user velocities and positions, and these variations could then have been removed from the pseudorange rate measurements. Similarly, fitting of low order polynomials can be used as suggested by de Bakker et al. (2009).

Filtering was preferred, in order to remove any reliance on external data such as satellite ephemerides: With filtering, only the actual measurements are employed and no other data, which could introduce additional errors, are used.

\section{Measurement validation}

In this section, the second experimental setup detailed in Fig. 1 has been used to further validate the presence of oscillatory components in the Galileo pseudorange rates.

In particular, the PSDs of the filtered measurements obtained from the two Galileo receivers are provided in Fig. 5. Data were collected from the roof-top antenna of the EMSL on GPS week 1755. Although the measurements from the Javad Delta receiver are noisier than the ones from the Septentrio PolarRxS receiver, a periodic component can be clearly identified in the measurements from Galileo IOV PFM and Galileo IOV FM2.

The PSDs clearly show the presence of oscillatory components affecting the measurements from Galileo IOV PFM and Galileo IOV FM2. The frequency and the period of the oscillations are 
Fig. 3 Pseudorange rate observables filtered to remove polynomial variations.

Oscillatory components can be seen in the measurements from Galileo IOV PFM and Galileo IOV FM2


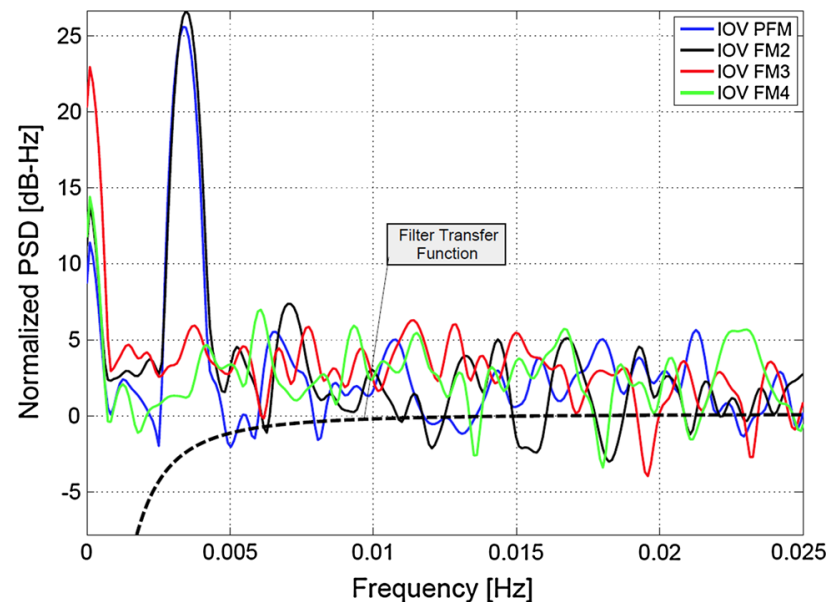

Fig. 4 PSDs of the filtered pseudorange rate measurements shown in Fig. 3. The PSDs have been normalized in order to give signals with unitary total power. The transfer function of the filter used for preprocessing pseudorange rate observations is also shown. $K=3$, $k_{\alpha}=0.99$. Given the good quality of the measurement, the Butterworth filter was not used

$f_{0}=0.0014 \mathrm{~Hz} \quad T_{0}=714 \mathrm{~s}$

The data used for the evaluation of the PSDs in Fig. 5 indicate that the oscillatory components observed in the measurements from Galileo IOV PFM and Galileo IOV FM2 have a time-varying centre frequency. This fact clearly emerges by comparing the results obtained in Fig. 4. Also in this case, no such component was observed in the data from Galileo IOV FM3 and Galileo IOV FM4.

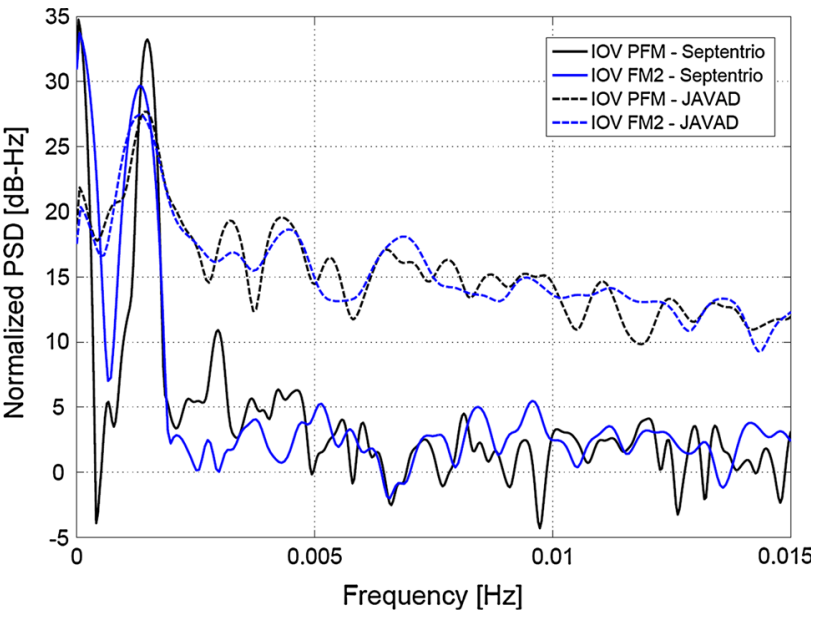

Fig. 5 PSDs of the filtered pseudorange rate measurements from the Septentrio and Javad receivers. The measurements are affected by the same oscillatory component

In addition to the use of a modified experimental setup, data were downloaded from the IGS (Dow et al. 2009) and used for the analysis. In this way, it was possible to use data from a different site, obtained in a different way. Station WTZ3 located in Wettzell, Germany, is equipped with a Javad Delta receiver and provides high data rate $(1 \mathrm{~Hz})$ Galileo observables in RINEX 3 format. These data were used to further verify the results described above.

The processing described above was applied to the data obtained from the WTZ3 station, and the findings presented below were obtained. The filtered measurements from 
Fig. 6 Pseudorange rate observables filtered using the pre-filtering stage to remove polynomial variations. Measurements from IGS station WTZ3, data collected on September 9, 2013
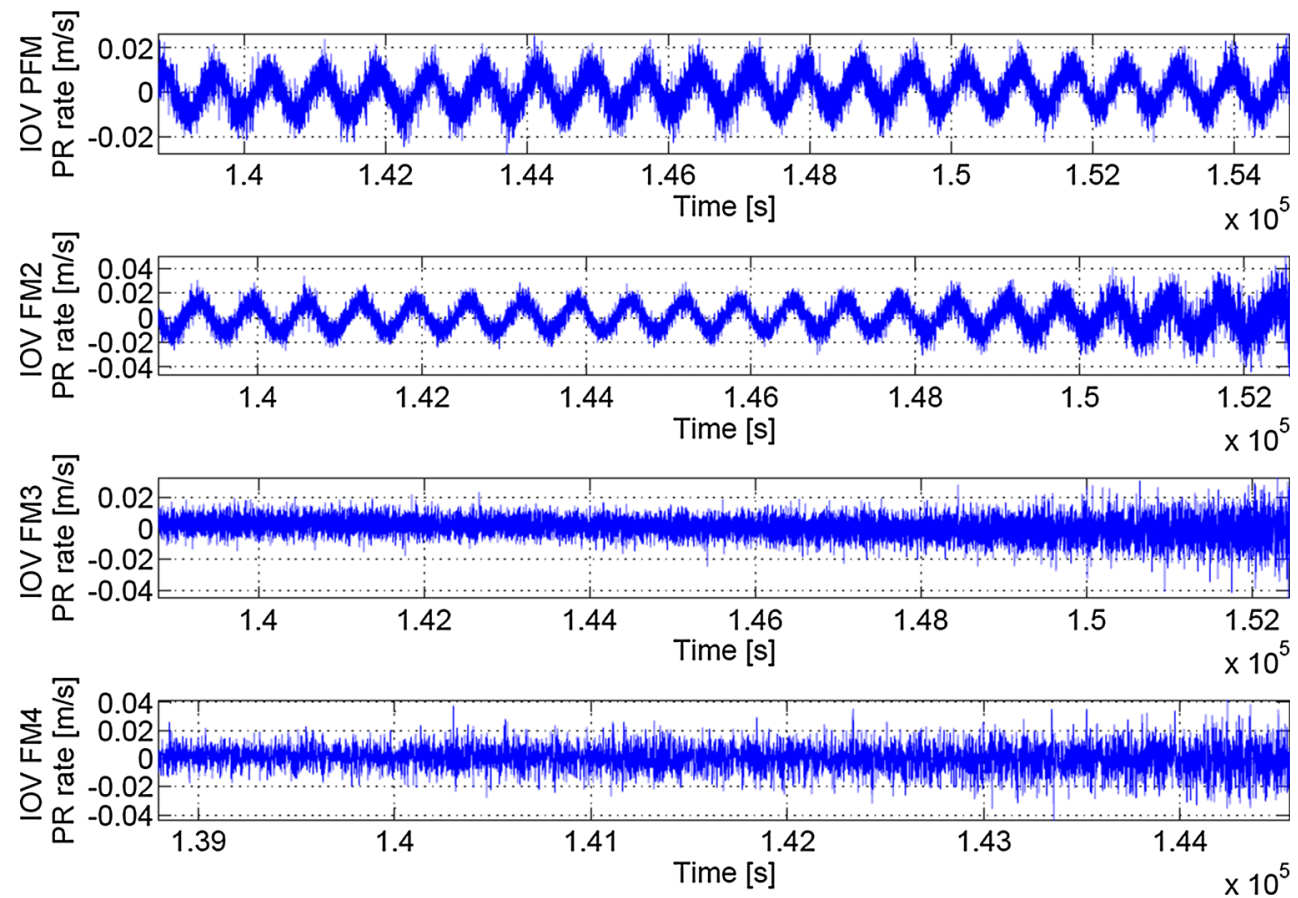

WTZ3 are provided in Fig. 6. Also in this case, an oscillatory component can be seen in the measurements from Galileo IOV PFM and Galileo IOV FM2.

The measurements taken at station WTZ3 are compared with that obtained at the JRC in Fig. 7 which shows the PSDs of the filtered pseudorange rate observations. The measurements were taken at the same time epochs and show similar spectral contents: The same phenomena are observed at the two sites. In this case, the oscillations on the measurements from Galileo IOV PFM and Galileo IOV FM2 have slightly different centre frequencies:

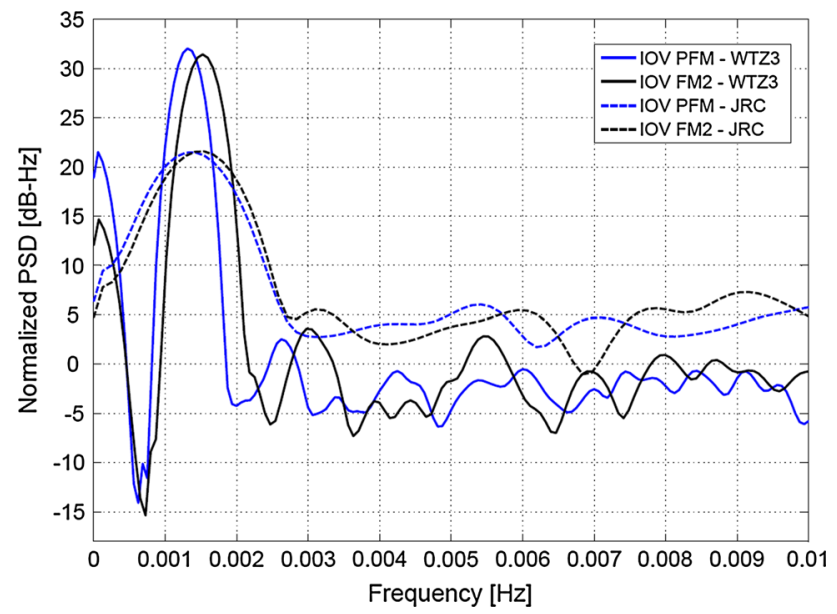

Fig. 7 PSDs of the filtered pseudorange rate measurements from the Septentrio receiver at the JRC site and from the Javad Delta receiver in Wettzell, Germany. The measurements were taken at the same time epochs and are affected by the same oscillatory component $f_{0,11}=0.00131 \mathrm{~Hz}, \quad f_{0,12}=0.00152 \mathrm{~Hz}$

These results confirm the findings obtained in the previous sections: Pseudorange rate measurements from Galileo IOV PFM and Galileo IOV FM2 are affected by oscillatory components which slightly degrade the final velocity solution obtained using Galileo IOV signals.

\section{Filtered velocity and Galileo velocity accuracy}

As mentioned in the introduction, the oscillations measured in the pseudorange rates are caused by the initial architecture adopted for combining the frequency references provided by the two active clocks present in the Galileo satellites. The combining algorithm has been improved in Galileo IOV FM3 and Galileo IOV FM4, and the oscillatory component is no longer present. Since the problem has been effectively solved, Galileo will be able to provide clean pseudorange rate measurements and improved velocity solutions.

The procedure detailed in the "Experimental setup and velocity computation" section was used for the computation of the receiver velocity. The analysis was performed on data from different GPS weeks. In all datasets considered, small oscillations, of the order of a few centimeters per second, were observed. Sample results are provided in Fig. 8, showing measurements from GPS week 1767: Oscillations are clearly visible on the East, North, and Up components of the velocity solution. Data were collected with $1 \mathrm{~Hz}$ rate from the antenna located on the roof-top of 
Fig. 8 Velocity solution obtained for a static receiver using the measurements from the four Galileo IOVs. Small oscillations (a few $\mathrm{cm} / \mathrm{s})$ are observable
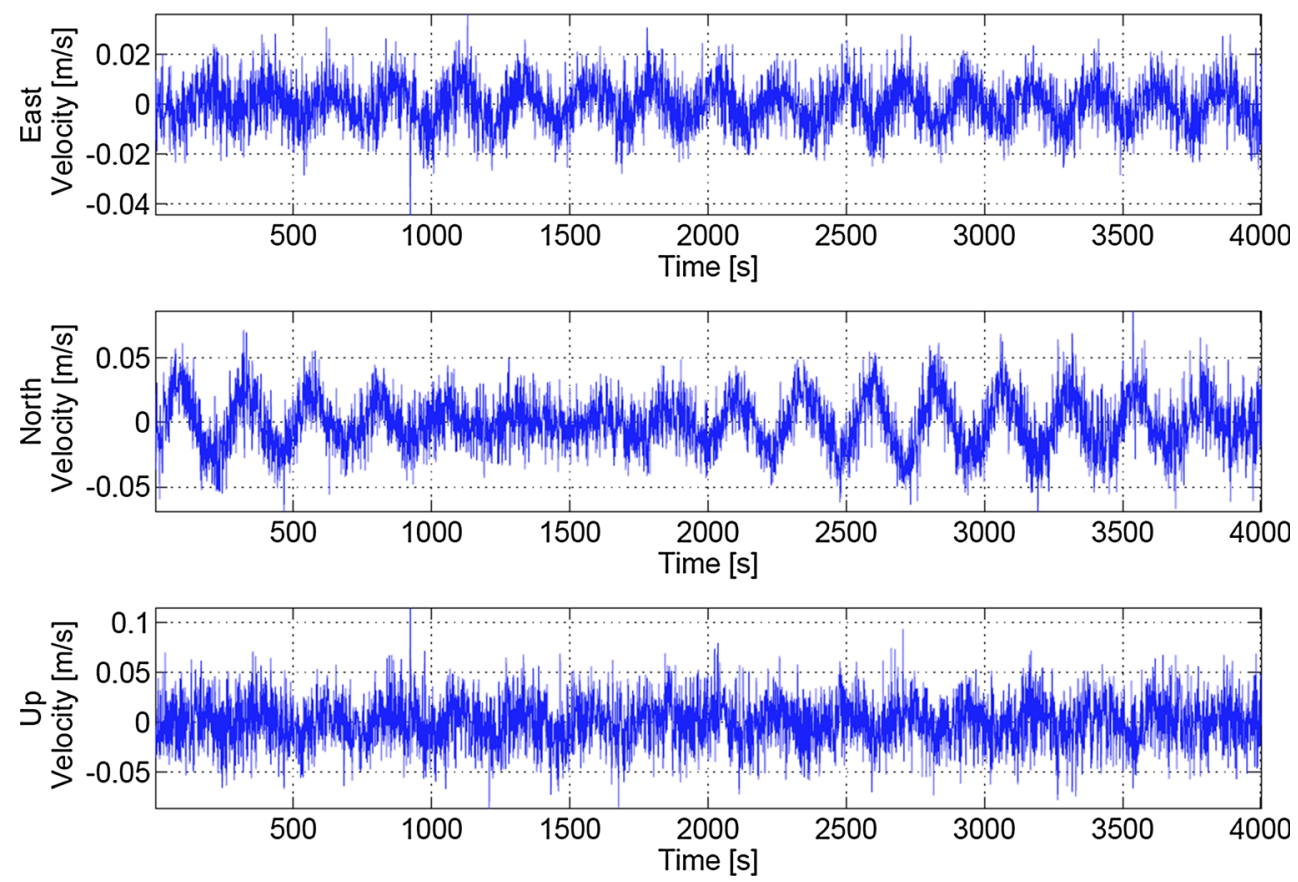

the EMSL $\left(45.81^{\circ} \mathrm{N}, 8.63^{\circ} \mathrm{E}, 279 \mathrm{~m}\right)$ using the Septentrio PolarRxS receiver. Hence, the impact of oscillations identified in the pseudorange rate can be clearly seen in Fig. 8.

The presence of an oscillatory term in the velocity solution is better highlighted in Fig. 9 which shows the PSDs of the three velocity components. Such oscillations will not be present in the velocity solutions computed using the signals from future Galileo satellites. Thus, they should be removed for the analysis of the final Galileo performance.

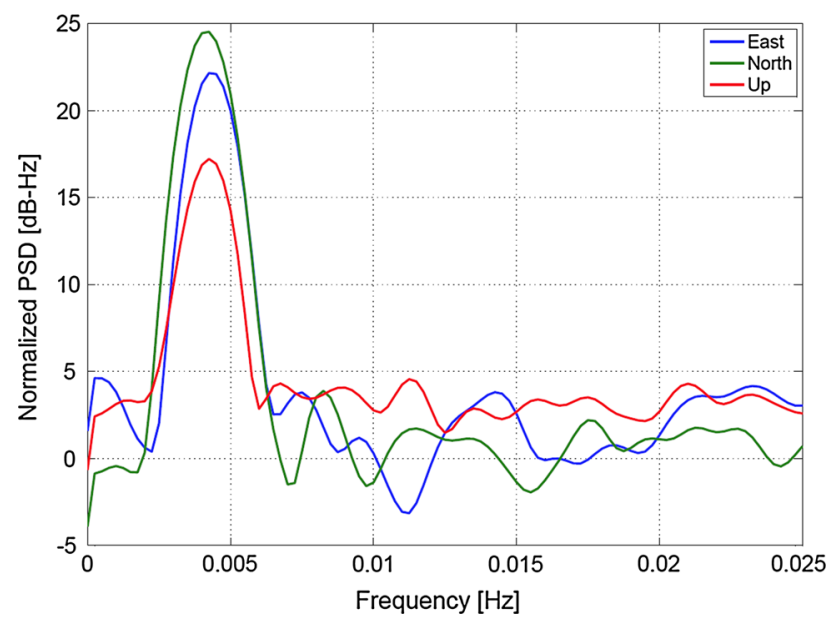

Fig. 9 PSDs of the three components of the velocity solution considered in Fig. 8. The presence of oscillatory terms appears clearly in all three velocity components. The PSDs have been normalized in order to give signals with unitary total power
The velocity accuracy that the Galileo system will be able to provide can be evaluated by filtering the oscillatory disturbance from the velocity solution obtained using the measurements currently available. In particular, the approach described in Fig. 10 has been adopted. Each velocity component is treated independently, and the PSD of each term is used to estimate the frequency, $f_{0}$, of the oscillatory disturbance.

The components of the velocity vector are separately filtered using the two pole notch filter (Borio et al. 2008) characterized by the following transfer function

$H_{n 2}(z)=\frac{1-2 \cos \left(2 \pi f_{0} T_{s}\right) z^{-1}+z^{-2}}{1-2 k_{\alpha} \cos \left(2 \pi f_{0} T_{s}\right) z^{-1}+k_{\alpha}^{2} z^{-2}}$

where $f_{0}$ is the frequency of the oscillatory disturbance, and $T_{\mathrm{s}}=1 / f_{\mathrm{s}}$ is the inverse of the measurement rate, $f_{\mathrm{s}}$. In this case, $f_{\mathrm{s}}=1 \mathrm{~Hz}$ and $k_{\alpha}$ is the pole contraction factor which was set to 0.97 . This value was selected in order to obtain a frequency notch sufficiently large to filter out the oscillatory component. Larger values of $k_{\alpha}$ do not allow the complete removal of the oscillatory disturbance. Note that

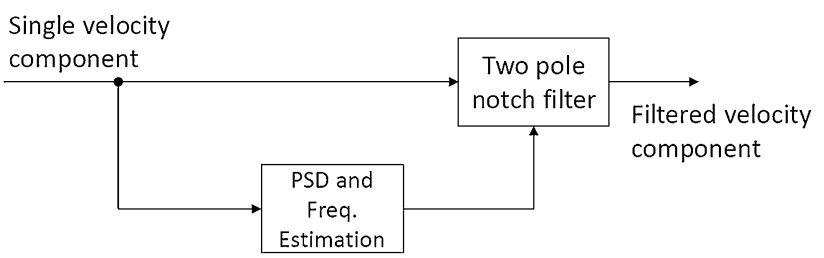

Fig. 10 Approach adopted for removing oscillatory disturbances from the Galileo velocity solution 
the notch filter used for removing the oscillatory component can introduce small distortions on the useful velocity signals and residual noise components. In order to assess the impact of such distortions, the PSDs of the filtered and unfiltered velocity components were analyzed. It was verified that the notch filter affects a frequency region which corresponds to approximately $2.6 \%$ of the total frequency range considered. This region corresponds to the oscillatory component identified. These distortions can thus be neglected for the analysis of the residual errors.

The notch filter allows the removal of the oscillatory component from the velocity vector without significantly amplifying the noise components present in the pseudorange rate measurements, as shown in Fig. 11.

The effect of filtering can be clearly seen in Fig. 11 where the three velocity components are separately plotted as a function of time. Filtering is effective in removing the oscillatory component which is no longer present. The statistics of the residual errors present in the Galileo velocity solutions are summarized in Table 1 for the filtered and unfiltered solutions. Performance has been evaluated in terms of root mean square (RMS), mean and maximum errors for both horizontal and vertical components.
It is worth noting that Gioia et al. (2014) have already evaluated the velocity solution using Galileo observables without filtering. Their results were affected by the oscillations defined in this research, but the cause was not identified.

In order to better understand the potential of Galileo, the performance of the filtered Galileo velocity solution was compared with that obtained using GPS. In order to perform a fair comparison between GPS and Galileo, similar geometry conditions were considered, and the GPS satellite geometry was artificially degraded. In particular, for each time epoch, four GPS satellites were selected in order to obtain a horizontal dilution of precision (HDOP) as close as possible to that obtained using the four IOV satellites. This selection process was performed using an exhaustive search approach: At each epoch, $\left(\begin{array}{c}N \\ 4\end{array}\right)$ subsets of four satellites were formed using the $N$ GPS satellites available. For each quartet, the algorithm developed computed the HDOP. The set with the HDOP the closest to that of the Galileo IOVs is used for the comparison with Galileo. Hence, a fair comparison between the two systems is possible for the horizontal component. This is the same
Fig. 11 Comparison between unfiltered and filtered velocity components. The oscillatory component is removed by filtering
Table 1 Statistics of the filtered and unfiltered Galileo velocity errors
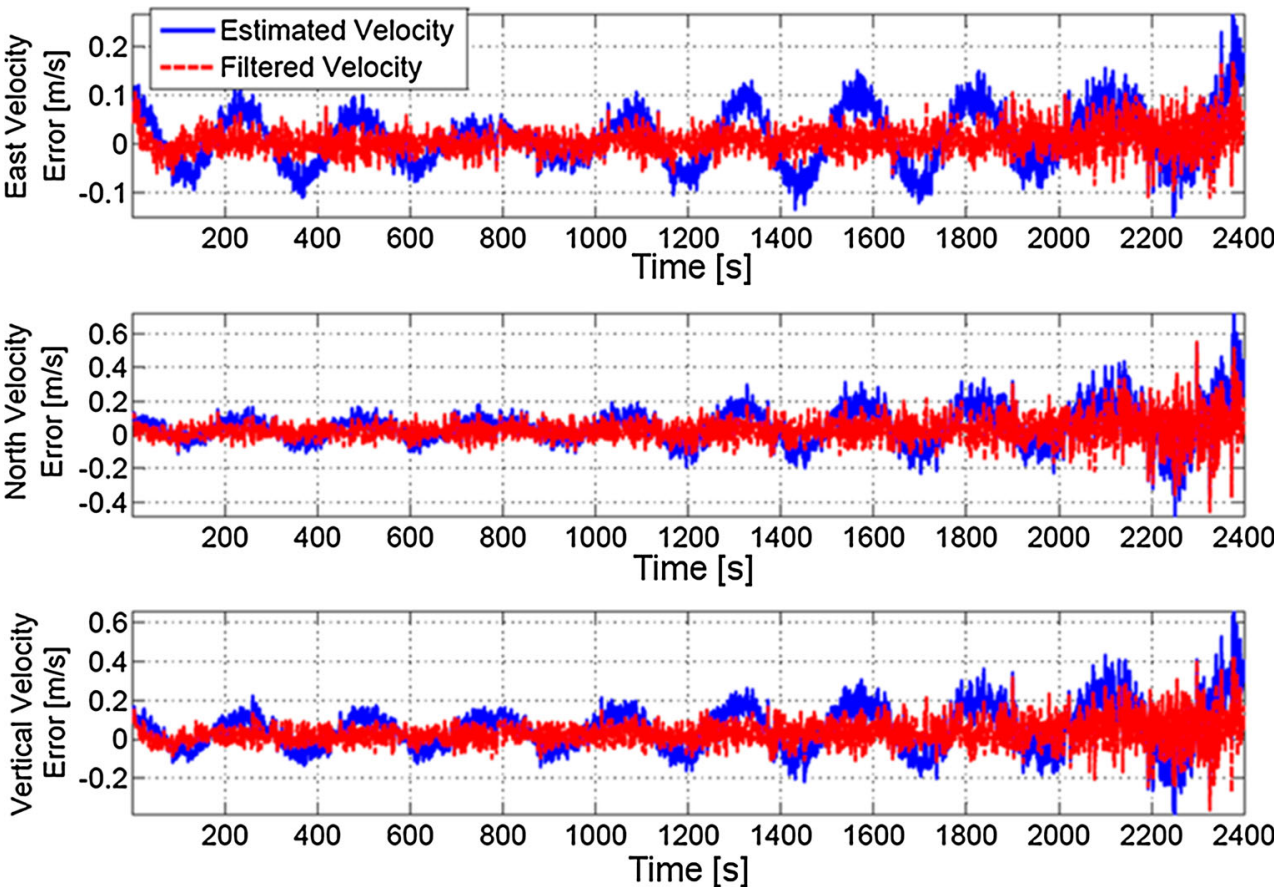

\begin{tabular}{|c|c|c|c|c|c|c|}
\hline \multirow[t]{2}{*}{ Configuration } & \multicolumn{2}{|l|}{$\operatorname{RMS}(\mathrm{m} / \mathrm{s})$} & \multicolumn{2}{|l|}{ Mean $(\mathrm{m} / \mathrm{s})$} & \multicolumn{2}{|l|}{$\operatorname{Max}(\mathrm{m} / \mathrm{s})$} \\
\hline & Horizontal & Vertical & Horizontal & Vertical & Horizontal & Vertical \\
\hline Galileo unfiltered & 0.1318 & 0.1260 & 0.1040 & 0.0529 & 0.7705 & 0.6589 \\
\hline Galileo filtered & 0.0840 & 0.0726 & 0.0626 & 0.0300 & 0.5609 & 0.4229 \\
\hline
\end{tabular}




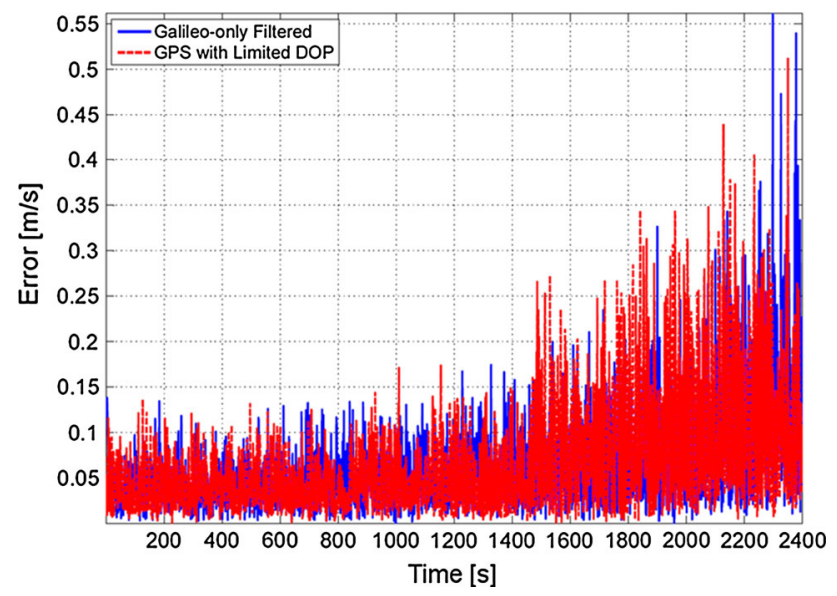

Fig. 12 Horizontal velocity errors of GPS with limited DOP and Galileo only filtered solutions

Table 2 Statistics of the velocity errors: comparison between GPS (with limited DOP) and filtered Galileo solutions

\begin{tabular}{llll}
\hline Configuration & $\begin{array}{l}\text { RMS } \\
\text { horizontal } \\
\text { error }(\mathrm{m} / \mathrm{s})\end{array}$ & $\begin{array}{l}\text { Mean } \\
\text { horizontal } \\
\text { error }(\mathrm{m} / \mathrm{s})\end{array}$ & $\begin{array}{l}\text { Maximum } \\
\text { horizontal error } \\
(\mathrm{m} / \mathrm{s})\end{array}$ \\
\hline $\begin{array}{l}\text { GPS limited } \\
\text { DOP }\end{array}$ & 0.0907 & 0.0671 & 0.5124 \\
$\begin{array}{l}\text { Galileo } \\
\text { filtered }\end{array}$ & 0.0840 & 0.0626 & 0.5609 \\
\hline
\end{tabular}

approach adopted in (Gioia et al. 2014) to compare Galileo and GPS position, velocity, and time (PVT) solutions. The results obtained are shown in Fig. 12.

From the figure, it emerges that the configurations have similar performance: The red dashed line and the blue curve are nearly coincident. The error statistics of the two configurations are summarized in Table 2.

The values provided in Table 2 confirm that Galileo and GPS have similar performance in the velocity domain. Although the maximum error observed for Galileo is higher than the GPS one, RMS and mean horizontal errors are slightly reduced. As shown in (Gioia et al. 2014), without filtering, Galileo performance seems degraded with respect to GPS.

\section{Conclusions}

We proposed a methodology for isolating oscillatory components in pseudorange rate observations and used it for the analysis of Galileo observables. From the analysis, it emerged that measurements from Galileo IOV PFM and Galileo IOV FM2 are affected by small oscillatory components which are due to the approach adopted for combining the reference signals provided by the Galileo on-board clocks. The problem has been solved, and measurements from the second pair of IOV satellites are not affected by such oscillations. Future Galileo satellites will therefore provide enhanced measurements and improved velocity solutions.

A notch filter was adopted to remove the oscillatory components from the Galileo velocity solution and assess the performance that Galileo will be able to achieve. The analysis shows that Galileo has performance similar to that of GPS under similar geometry conditions.

Future work will include the investigation of other Galileo observables and the impact of the oscillations identified on such measurements.

Open Access This article is distributed under the terms of the Creative Commons Attribution License which permits any use, distribution, and reproduction in any medium, provided the original author(s) and the source are credited.

\section{References}

Benton CJ, Mitchell CN (2012) GPS satellite oscillator faults mimicking ionospheric phase scintillation. GPS Solut 16(4): 477-482. doi:10.1007/s10291-011-0247-3

Benton CJ, Mitchell CN (2014) Further observations of GPS satellite oscillator anomalies mimicking ionospheric phase scintillation. GPS Solut 18(3):387-391. doi:10.1007/s10291-013-0338-4

Borio D, Camoriano L, Mulassano P (2006) Analysis of the one-pole notch filter for interference mitigation: Wiener solution and loss estimations. In: Proceedings of ION GNSS 2006, Institute of Navigation, Fort Worth, September, 1849-1860

Borio D, Camoriano L, Lo Presti L (2008) Two-pole and multi-pole notch filters: a computationally effective solution for GNSS interference detection and mitigation. IEEE Syst J 2(1):38-47. doi:10.1109/ITST.2007.4295837

de Bakker PF, van der Marel H, Tiberius CCJM (2009) Geometryfree undifferenced, single and double differenced analysis of single frequency GPS, EGNOS and GIOVE-A/B measurements. GPS Solut 13(4):305-314. doi:10.1007/s10291-009-0123-6

Dow JM, Neilan RE, Rizos C (2009) The International GNSS service in a changing landscape of global navigation satellite systems. J Geod 83(3-4):191-198. doi:10.1007/s00190-008-0300-3

Gioia C, Borio D, Angrisano A, Gaglione S, Fortuny-Guasch J (2014) A Galileo IOV assessment: measurement and position domain. GPS Solut. doi:10.1007/s10291-014-0379-3

Hoffmann-Wellenhof B, Lichtenegger H, Collins J (1992) Global positioning system: theory and practice. Springer, New York

Kaplan ED, Hegarty J (2006) Understanding GPS: principles and applications, 2nd ed. Artech House Mobile Communications Series, Norwood, MA

Odijk D, Teunissen PJG, Khodabandeh A (2014) Galileo IOV RTK positioning: standalone and combined with GPS. Surv Rev 46(337):267-277. doi:10.1179/1752270613Y.0000000084

Proakis JG, Manolakis DK (1995) Digital signal processing: principles, algorithms and applications, 3rd edn. Prentice Hall, Englewood Cliffs

Rochat P, Droz F, Wang Q, Froidevaux S (2012) Atomic clocks and timing systems in global navigation satellite systems. In: Proceedings of European navigation conference, Gdansk, April $1-11$

Stoica P, Moses R (2005) Spectral analysis of signals. Prentice Hall, Englewood Cliffs 

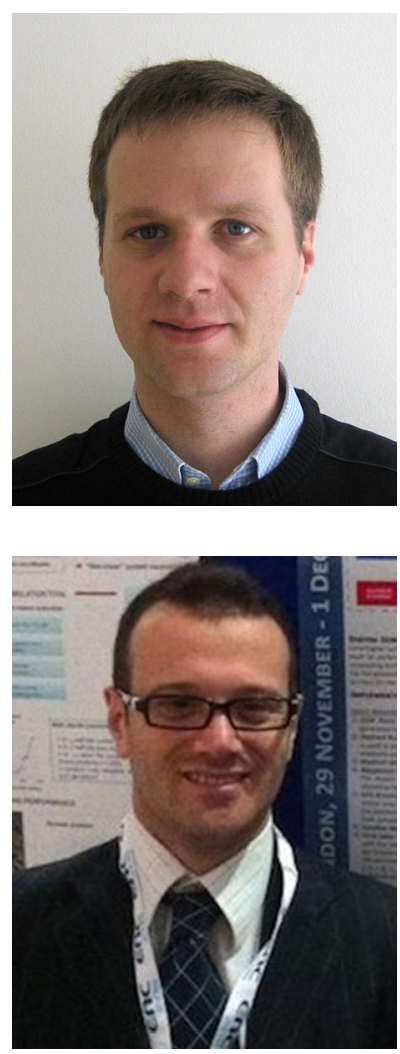

Daniele Borio received MS and $\mathrm{PhD}$ from Politecnico di Torino in 2004 and 2008, respectively. From January 2008 to September 2010, he was a senior research associate in the PLAN group of the University of Calgary, Canada. He is currently a scientific and policy officer at the Joint Research Centre of the European Commission in the fields of digital and wireless communications, location and navigation.

Ciro Gioia received MS in Nautical Sciences from Parthenope University, Italy, in 2009. In April 2014, he successfully defended his $\mathrm{PhD}$ thesis at the same University. From May 2013 to April 2014, he was a visiting student at the European Commission Joint Research Centre (JRC). Since May 2014, he is external consultant at JRC. His research interest focuses on location and navigation with special emphasis on geomatics aspects.

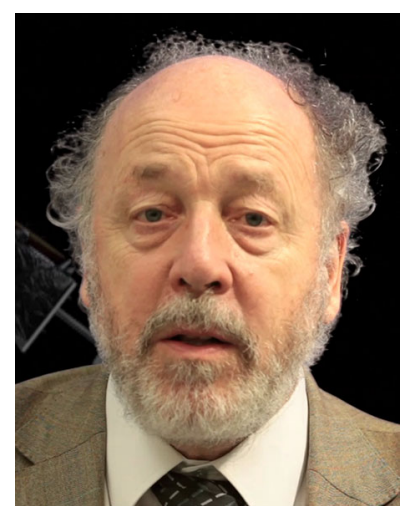

Neil Mitchison's original background is in mathematics and computing. For most of the last 25 years, he has been working in risk and dependability analysis in a wide range of domains. From 2012 to 2014, he was Head of the "Security Technology Assessment" unit of the Joint Research Centre of the European Commission, taking strategic charge of a group of researchers studying the dependability and the vulparticular the nascent Galileo system. nerabilities of GNSSs, in 